\title{
Channel Estimation Scheme for the Enhanced Reliability in the Flying Ad-hoc Network
}

\author{
Sang-Young Kim, Jae-Hyun Ro, Hyoung-Kyu Song* \\ uT Communication Research Institute, Sejong University, Seoul, Korea (E-mail: 12ksy12@naver.com) \\ uT Communication Research Institute, Sejong University, Seoul, Korea (E-mail: ilovebisu@nate.com) \\ uT Communication Research Institute, Sejong University, Seoul, Korea (E-mail: songhk@sejong.ac.kr) \\ * Corresponding Author's E-mail : songhk@ sejong.ac.kr
}

\begin{abstract}
This paper proposes the improved channel estimation for flying ad-hoc network (FANET). The FANET means the wireless communication network between the unmanned aerial vehicle (UAV). UAVs have unlimited availability not only in war but in various fields such as reconnaissance, observation, exploration. Due to the high-mobility of the UAVs, these channels of the FANET environment are very dynamic and time varying channel. Because the FANET for UAV transfers data such as the information on mission accomplishment, collision prevention, etc., the FANET requires the robust and spectral efficient communication scheme in the fading channel. IEEE $802.11 \mathrm{p}$ is considered as a communication method between UAVs. However, IEEE $802.11 \mathrm{p}$ suffers from the challenge of robust channel estimation due to rapid time-varying nature of the channel. In the IEEE $802.11 \mathrm{p}$, the long training symbols are used for fine synchronization and channel estimation. This channel estimation scheme isn't suitable in the time varying channel. This paper proposes the scheme that uses the pilot symbols and recovered symbols to estimate channel. The proposed scheme can provide the reliable wireless communication for the FANET.
\end{abstract}

Keywords - channel estimation, flying ad-hoc network, $802.11 \mathrm{p}$

\section{INTRODUCTION}

Recently, unmanned aerial vehicle (UAV) that has unlimited availability not only in war but in various fields such as reconnaissance, observation, exploration has been studied. Accordingly, the flying ad-hoc network (FANET) which is communication network between UAVs is important. FANET has higher dynamic channel due to rapidly varying environments. Additionally, FANET requires the wide coverage of the wireless communication. IEEE $802.11 \mathrm{p}$ is considered as a wireless communication for the FANET. IEEE 802.11p standard is a wireless communication standard for outdoor applications. IEEE $802.11 \mathrm{p}$ based on IEEE 802.11a standard was developed [1]. Because IEEE 802.11a is the standard for stationary indoor environments, IEEE $802.11 \mathrm{p}$ has several issues in high-mobility, fast time varying wireless communication environments [2].

Figure 1 shows the FANET environment. The FANET is used for the information exchange such as the information on mission accomplishment, collision prevention, etc. between UAVs. Therefore, the FANET should provide the reliable wireless communication.

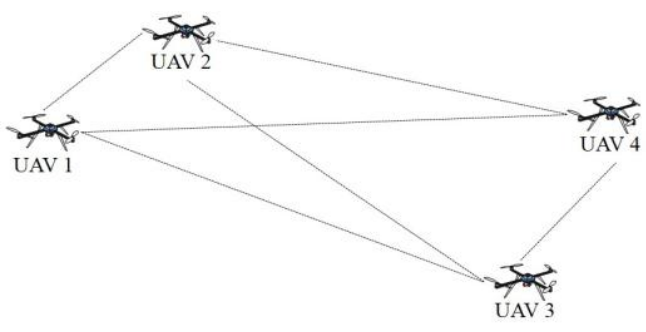

Fig 1: FANET environment

The IEEE 802.11p uses the only long training symbols for the channel estimation. However, the channel estimated by using long preamble may not be valid for all of the OFDM symbols within a frame leading to severe intersymbol-interference (ISI) and the worst performance in fast time varying channels [3][5]. This paper proposes the improved channel estimation scheme for the FANET in time varying channel.

This paper is organized as follows. The section 2 explains the IEEE 802.11p standard for FANET communication. The section 3 explains the proposed scheme in detail. In the section 4, the simulation results are explained. Finally, the section 5 is the conclusion. 


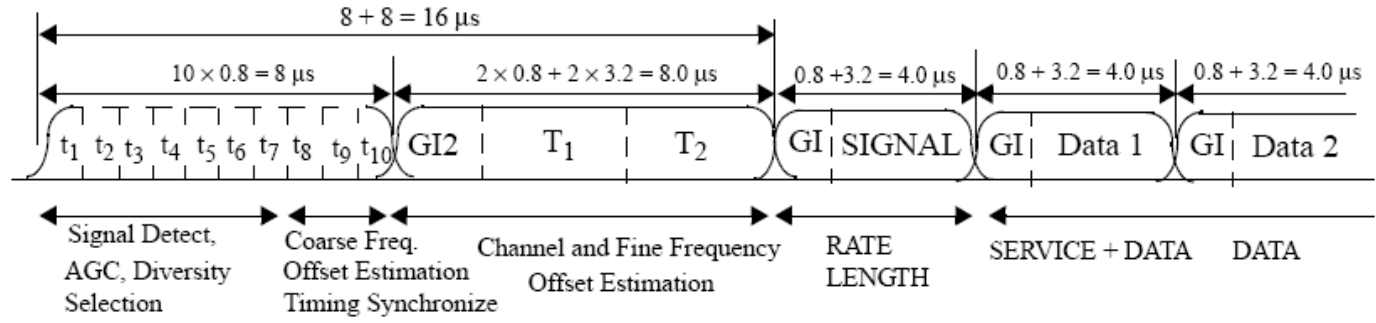

Fig 2: Packet preamble structure of IEEE 802.11p

\section{IEEE 802.11P FOR FANET}

Table 1 shows the parameters of the IEEE 802.11p. IEEE 802.11p uses the orthogonal frequency division multiplexing (OFDM) and is based on IEEE 802.11a. The transmitter uses a convolutional encoder for forward error correction. The 64-point inverse fast Fourier transform (IFFT) is used for the realization of the OFDM modulation. 48 OFDM subcarriers are used as the data subcarriers and 4 OFDM subcarriers are used as the pilot subcarriers. 12 OFDM subcarriers are used as the guard interval (GI). IEEE $802.11 \mathrm{p}$ uses $10 \mathrm{MHz}$ as the system bandwidth. $10 \mathrm{MHz}$ is the half of the bandwidth of the IEEE 802.11a. Therefore, the IEEE $802.11 p$ provides the half bit rate of the bit rate of IEEE 802.11a. However, the symbol period of IEEE $802.11 \mathrm{p}$ is twice the symbol period of IEEE 802.11a. As a result, the GI duration is extended. The extended GI duration mitigates the effect of the large delay spread of the channel and offers increased robustness.

Table 1: Parameters of the IEEE 802.11p

\begin{tabular}{|l|l|}
\hline \multicolumn{1}{|c|}{ Parameters } & \multicolumn{1}{c|}{ Value } \\
\hline Bit rate (Mbps) & $\begin{array}{l}\text { 3, 4.5, 6, 9, 12, 18, 24, } \\
27\end{array}$ \\
\hline Modulation mode & $\begin{array}{l}\text { BPSK, QPSK, 16QAM, } \\
\text { 64QAM }\end{array}$ \\
\hline Code rate & $1 / 2,2 / 3,3 / 4$ \\
\hline Number of subcarriers & 52 \\
\hline Bandwidth & $10 \mathrm{MHz}$ \\
\hline Symbol duration & $8 \mu \mathrm{m}$ \\
\hline Guard time & $1.6 \mu \mathrm{m}$ \\
\hline FFT period & $6.4 \mu \mathrm{m}$ \\
\hline Preamble duration & $32 \mu \mathrm{m}$ \\
\hline Subcarrier spacing & $0.15625 \mathrm{MHz}$ \\
\hline
\end{tabular}

Figure 2 shows the $802.11 \mathrm{p}$ packet preamble structure. The preamble is consist of the short training symbols, the long training symbols, the signal field and the data field. The short training symbols are used for coarse synchronization and the long training symbols are used for fine synchronization and channel estimation. The information of the modulation type, the code rate and the length of the packet is conveyed through the signal field. The data field conveys the data.

\section{PROPOSED SCHEME}

In this section, the proposed scheme is explained in detail. The IEEE 802.11p uses the only long training symbols for fine synchronization and channel estimation. This scheme is not suitable in the FANET. This paper proposes the improved channel estimation scheme.

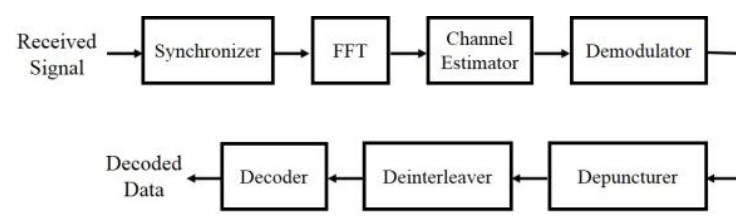

Fig 3: Structure of the receiver

Figure 3 shows the structure of the receiver. The receiver conducts the channel estimation in the channel estimator. In the FANET, the channel has the extremely dynamic characteristic. The proposed scheme uses the characteristic that the correlation of the channel response (CR) between the adjacent two data symbol is high. In this paper, the least squares (LS) scheme is used in initial channel estimation. The estimated CR is as follows,

$$
H_{L S}(k)=\frac{R_{T_{1}}(k)+R_{T_{2}}(k)}{2 X(k)} .
$$

Table 2: Parameters of time varying channel

\begin{tabular}{|l|l|l|l|l|l|}
\hline Column Index & $\begin{array}{c}\text { Tap } \\
\text { Power }(\mathbf{d B})\end{array}$ & $\begin{array}{c}\text { Relative } \\
\text { Path Loss (dB) }\end{array}$ & $\begin{array}{c}\text { Delay } \\
\text { Value (ns) }\end{array}$ & $\begin{array}{c}\text { Fading } \\
\text { Doppler (Hz) }\end{array}$ & $\begin{array}{c}\text { Channel } \\
\text { Type }\end{array}$ \\
\hline 1 & 0.0 & 0.0 & 0 & 60 & Rician \\
\hline 2 & & -24.9 & 1 & 858 & Rayleigh \\
\hline 3 & & -25.5 & 2 & 486 & Rayleigh \\
\hline
\end{tabular}



ISSN : 2248-9622, Vol. 7, Issue 4, ( Part -4) April 2017, pp.63-66

\begin{tabular}{|l|l|l|l|l|l|}
\hline 4 & -6.3 & -13.1 & 100 & 655 & Rayleigh \\
\hline 5 & & -7.5 & 101 & 56 & Rayleigh \\
\hline 6 & -25.1 & -28.9 & 200 & 823 & Rayleigh \\
\hline 7 & & -29.3 & 201 & 75 & Rayleigh \\
\hline 8 & & -35.6 & 202 & 99 & Rayleigh \\
\hline 9 & -22.7 & -25.7 & 300 & 110 & Rayleigh \\
\hline 10 & & -34.4 & 301 & 639 & Rayleigh \\
\hline 11 & & -27.4 & 302 & 858 & Rayleigh \\
\hline
\end{tabular}

$X(k)$ is the initial CR. The $k$ is sub-carrier index.

The $R_{T_{1}}(k)$ and $R_{T_{2}}(k)$ are the two training symbols. After the initial channel estimation, the characteristic that the correlation of the $\mathrm{CR}$ between the adjacent two data symbols is high is used in the channel estimation. The step of the channel estimation is as follows.

In the first step, the estimated $\mathrm{CR} \hat{S}_{T, i}(k)$ is obtained. The $i$ is symbol index.

$$
\hat{S}_{T, i}(k)=\frac{S_{R, i}(k)}{H_{C E, i-1}(k)} \text {. }
$$

The $S_{R, i}(k)$ is the received signal and the $H_{C E, i-1}(k)$ is the previous CR. The $\hat{S}_{T, i}(k)$ is demapped to the data symbol $\hat{X}_{i}(k)$. The $\hat{X}_{i}(k)$ is available for the channel estimation.

In the next step, the $\hat{S}_{T, i}(k)$ and $\hat{X}_{i}(k)$ are used in the estimation. The estimated $\mathrm{CR} \hat{H}_{i}(k)$ is as follows,

$$
\hat{H}_{i}(k)=\frac{S_{R, i}(k)}{\hat{X}_{i}(k)} .
$$

The $H_{i}(k)$ is used to estimate the data symbol that can obtain through the equation (4).

$$
H_{i}(k)=\frac{1}{2} H_{i-1}(k)+\frac{1}{2} \hat{H}_{i}(k) .
$$

The $H_{i}(k)$ is used to estimate the data symbol. In the proposed scheme, all data and pilot sub-carriers of the data field are used for the channel estimation. The proposed scheme can provide the improved reliability in the FANET by using the characteristic that the correlation of the channel response (CR) between the adjacent two data symbol is high. The simulation results show that the proposed scheme has the better reliability than conventional channel estimation.

\section{SIMULATION RESULTS}

In this section, the simulation results are explained. In the simulation, the time varying channel is used as the simulation environment. Table 2 shows the parameters of the time varying channel [4]. The column index 1, 4, 6 and 9 are used in this simulation. The tap power means the reduced power of the transmitted signal. The fading Doppler means the value of Doppler frequency caused by the highmobility. Table 3 shows the transmission types of the IEEE 802.11p.

Table 3: Transmission types

\begin{tabular}{|c|l|l|l|}
\hline $\begin{array}{c}\text { Transmission } \\
\text { type }\end{array}$ & $\begin{array}{c}\text { Modulation } \\
\text { scheme }\end{array}$ & $\begin{array}{c}\text { Code } \\
\text { rate }\end{array}$ & $\begin{array}{c}\text { Data } \\
\text { rate } \\
\text { (Mbps) }\end{array}$ \\
\hline $\mathbf{1}$ & BPSK & $1 / 2$ & 3 \\
\hline $\mathbf{2}$ & BPSK & $3 / 4$ & 4.5 \\
\hline $\mathbf{3}$ & QPSK & $1 / 2$ & 6 \\
\hline $\mathbf{4}$ & QPSK & $3 / 4$ & 9 \\
\hline $\mathbf{5}$ & 16-QAM & $1 / 2$ & 12 \\
\hline $\mathbf{6}$ & 16-QAM & $3 / 4$ & 18 \\
\hline $\mathbf{7}$ & 64-QAM & $2 / 3$ & 24 \\
\hline $\mathbf{8}$ & 64-QAM & $3 / 4$ & 27 \\
\hline
\end{tabular}

Figure 4 and 5 show the comparison of the proposed and conventional scheme. The results are divided into two cases for the convenience. The solid lines mean the results of the proposed scheme and the dotted lines mean the results of the conventional scheme. Figure 4 shows the transmission type 1 to 4 of the proposed and conventional scheme. Figure 5 shows the transmission type 5 to 8 of the proposed and conventional scheme. Figure 4 and 5 show that the proposed scheme has better reliability than the conventional scheme. 


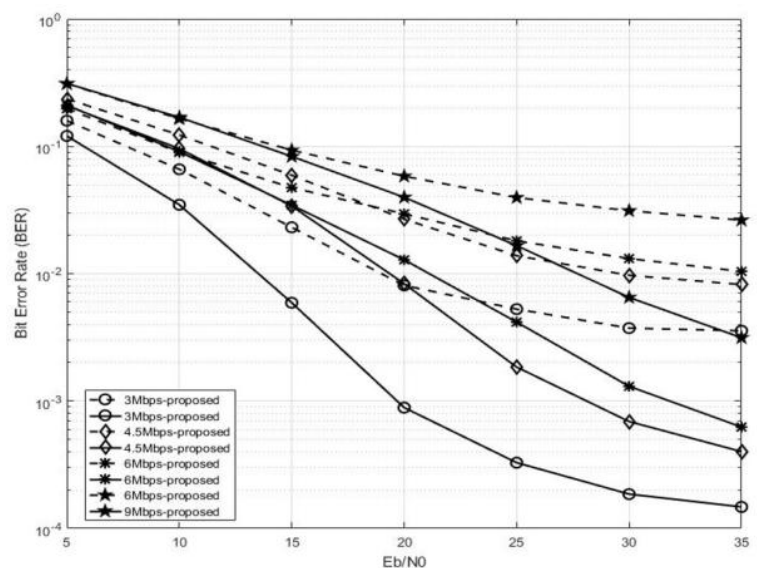

Fig 4: Comparison of proposed and conventional scheme

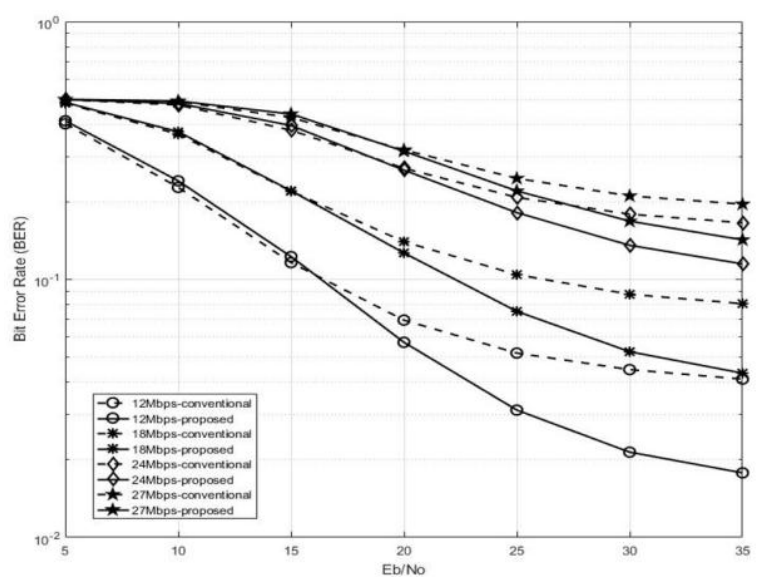

Fig 5: Comparison of proposed and conventional scheme

\section{CONCLUSION}

The proposed scheme uses the characteristic that the correlation of the CR between the adjacent two data symbols are high. Thus, the proposed scheme can provide the improved reliability for the FANET. The simulation results show that the proposed scheme has better reliability than the conventional scheme in the time varying channel. Therefore, the proposed scheme can usefully be used in the dynamic channel environment.

\section{ACKNOWLEDGEMENTS}

This research was supported by R\&D Program through the National Research Foundation of Korea(NRF) funded by the Ministry of Science, ICT \& Future Planning (NRF-2016M 1B3A1A02937594) (Corresponding Author: Hyoung-Kyu Song).

\section{REFERENCES}

[1] J.B. Kenney, 2011. Dedicated Short-Range Communication (DSRC) Standards in the United States. IEEE. VOL. 99, NO. 7, pp. 1162-1182

[2] A. Paier et al, 2010. Average Downstream Performance of Measured IEEE 802.11p Infrastructure-to-Vehicle Links. IEEE. ICC. pp. 1-5

[3] H.A. Hamid, K. Tepe, and E.A. Raheem, 2007. Performance of DSRC Systems Using Conventional Channel Estimation at High Velocities. Int. J. Electron. Commun. (AEU), VOL. 61, NO, 8, pp. 556-561

[4] Guillermo Acosta-Marum 2007. Measurement, Modeling, and OFDM Synchronization for the Wideband Mobile-toMobile Channel.

[5] Y.D. Lee, D.H. Park, and H.K. Song 2008. Improved Channel Estimation and MAIRobust Schemes for Wireless OFDMA System. pp. 213-223 